\title{
Twistor origin of the superstring
}

\section{Nathan Berkovits}

ICTP South American Institute for Fundamental Research, Instituto de Física Teórica, UNESP - Univ. Estadual Paulista, Rua Dr. Bento T. Ferraz 271, 01140-070, São Paulo, SP, Brasil

E-mail: nberkovi@ift.unesp.br

ABSTRACT: After introducing a $\mathrm{d}=10$ pure spinor $\lambda^{\alpha}$, the Virasoro constraint $\partial x^{m} \partial x_{m}=0$ can be replaced by the twistor-like constraint $\partial x^{m}\left(\gamma_{m} \lambda\right)_{\alpha}=0$. Quantizing this twistorlike constraint leads to the pure spinor formalism for the superstring where the fermionic superspace variables $\theta^{\alpha}$ and their conjugate momenta come from the ghosts and antighosts of the twistor-like constraint.

KeYwords: Superstrings and Heterotic Strings, BRST Quantization, Topological Strings

ARXiv EPRINT: 1409.2510 


\section{Contents}

1 Introduction 1

2 Worldsheet action 5

$\begin{array}{llr}3 & \text { Gauge invariances } & 6\end{array}$

$\begin{array}{lll}4 & \text { Gauge fixing } & 7\end{array}$

5 Gauge-fixed pure spinor formalism 9

6 Gauge-fixed non-minimal pure spinor formalism 10

$\begin{array}{lll}7 & \text { Generalization to curved backgrounds } & 11\end{array}$

\section{Introduction}

The conventional manner to obtain the superstring from the bosonic string is to generalize the worldsheet conformal invariance generated by the Virasoro constraint $\partial x^{m} \partial x_{m}=0$ to the worldsheet $\mathrm{N}=1$ superconformal invariance generated by the super-Virasoro constraint $\partial x^{m} \psi_{m}=0$ where $\psi^{m}$ is the fermionic worldsheet superpartner of $x^{m}$. This RamondNeveu-Schwarz (RNS) construction of the superstring [1, 2] was developed in the 1970's, and although it is based on the simple geometrical idea of generalizing conformal invariance to superconformal invariance, it has the disadvantage that spacetime supersymmetry is only present after including both periodic and antiperiodic conditions for $\psi^{m}$ and performing a GSO projection [3] which truncates out states constructed from an even number of $\psi^{m}$ variables. This lack of manifest spacetime supersymmetry makes it difficult to compute scattering amplitudes involving fermionic states and has prevented the RNS formalism from being used to describe Ramond-Ramond backgrounds.

In the 1980's, Green and Schwarz developed a new formalism $[4,5]$ for the superstring in which spacetime supersymmetry is manifest and is constructed using a spacetime spinor variable $\theta^{\alpha}$ intead of the spacetime vector variable $\psi^{m}$ of the RNS formalism. In addition to worldsheet conformal invariance, their superstring action contains a fermionic symmetry called "kappa symmetry" [6] which replaces the $\mathrm{N}=1$ worldsheet superconformal invariance of the RNS formalism. However, the structure of kappa symmetry prevented quantization of the Green-Schwarz formalism except in light-cone gauge, which complicates the computation of scattering amplitudes and the quantum description of Ramond-Ramond backgrounds.

Starting in 2000, a new formalism for the superstring has been developed in which spacetime supersymmetry is manifest and which can be easily quantized in a covariant 
manner [7]. In addition to the fermionic spinor variable $\theta^{\alpha}$ of the Green-Schwarz formalism, this new formalism includes a bosonic spinor variable $\lambda^{\alpha}$ which satisfies the $d=10$ "pure spinor" constraint

$$
\lambda \gamma^{m} \lambda=0
$$

for $m=0$ to 9 . Unlike the RNS and Green-Schwarz formalisms, it has been successfully used to compute multiloop amplitudes involving both bosonic and fermionic states [8-10] and to prove the quantum consistency of Ramond-Ramond backgrounds such as $A d S_{5} \times$ $S^{5}[11-13]$.

However, unlike the RNS formalism, the geometric origin of this new formalism was not understood. Physical states and scattering amplitudes are defined using a gauge-fixed action and a nilpotent BRST operator $Q$ constructed from the Green-Schwarz variables and the pure spinor $\lambda^{\alpha}$ as

$$
Q=\int d z \lambda^{\alpha} d_{\alpha}
$$

where $d_{\alpha}$ is the fermionic Green-Schwarz-Siegel constraint [14] which generates kappa symmetry. But despite several attempts [15-18], this pure spinor BRST operator was not obtained in a simple manner by gauge-fixing a worldsheet reparameterization invariant action.

In this paper, an elegant geometrical origin for this formalism will be proposed and the pure spinor BRST operator of (1.2) will be obtained by gauge-fixing a simple worldsheet reparameterization invariant action. Surprisingly, this reparameterization invariant action will be constructed entirely from bosonic worldsheet variables, and the fermionic worldsheet variables $\theta^{\alpha}$ and their conjugate momenta will come from ghosts and antighosts associated with the gauge fixing.

The bosonic variables in the worldsheet action will consist of the usual $\mathrm{d}=10$ spacetime vector variable $x^{m}$ together with a spacetime spinor variable $\lambda^{\alpha}$ satisfying the $\mathrm{d}=10$ pure spinor constraint $\lambda \gamma^{m} \lambda=0$. The pure spinor constraint implies that only 11 of the 16 components of $\lambda^{\alpha}$ are independent, and after Wick-rotation to Euclidean signature, $\lambda^{\alpha}$ parameterizes the eleven-dimensional complex space $\frac{\mathrm{SO}(10)}{\mathrm{U}(5)} \times C^{*}$ where $C^{*}$ is the complex plane minus the origin [19].

Instead of generalizing the Virasoro constraint $T=-\frac{1}{2} \partial x^{m} \partial x_{m}=0$ to a superVirasoro constraint as in the RNS formalism, the Virasoro constraint $T=0$ will instead by replaced by the twistor-like constraint

$$
C_{\alpha}=-\frac{1}{2} \partial x^{m}\left(\gamma_{m} \lambda\right)_{\alpha}=0
$$

Note that $C_{\alpha}=0$ implies $T=0$ since $T$ is equal to $\frac{1}{\lambda^{\beta} \bar{\lambda}_{\beta}} C_{\alpha}\left(\gamma_{m} \bar{\lambda}\right)^{\alpha} \partial x^{m}$ where $\bar{\lambda}_{\alpha}$ is any spinor satisfying $\lambda^{\alpha} \bar{\lambda}_{\alpha} \neq 0$. As discussed by several authors [20-29], pure spinors are the natural generalization to higher dimensions of $\mathrm{d}=4$ Penrose twistors [30], and (1.3) is the $\mathrm{d}=10$ stringy version [31-41] of the $\mathrm{d}=4$ twistor constraint $\left(\frac{\partial}{\partial \tau} x_{a \dot{a}}\right) \lambda^{a}=0$ where $a, \dot{a}=1$ to 2 and $x_{a \dot{a}}(\tau)$ is a $\mathrm{d}=4$ light-like trajectory. 
The worldsheet reparameterization invariant action for $x^{m}$ and $\lambda^{\alpha}$ will be

$$
S=\int d^{2} z(\operatorname{det} e)\left(\frac{1}{2} \nabla x^{m} \bar{\nabla} x_{m}+w_{\alpha} \bar{\nabla} \lambda^{\alpha}+L^{\alpha} C_{\alpha}+\lambda^{\alpha} \bar{\Lambda}_{\alpha}\right)
$$

where $\nabla=e_{-}^{J} \partial_{J}, \bar{\nabla}=e_{+}^{J} \partial_{J}, e_{ \pm}^{J}$ is the usual two-dimensional vierbein, and $L^{\alpha}$ is a Lagrange multiplier which enforces the constraint $C_{\alpha}=0$. In addition, the term $\lambda^{\alpha} \bar{\Lambda}_{\alpha}$ has been included in the Lagrangian where $\bar{\Lambda}_{\alpha}$ is a bosonic pure spinor of opposite chirality to $\lambda^{\alpha}$. If $\bar{\Lambda}_{\alpha}$ is interpreted (in Euclidean signature) as the complex conjugate to $\lambda^{\alpha}$, the term $\lambda^{\alpha} \bar{\Lambda}_{\alpha}$ concentrates the functional integration over $\lambda^{\alpha}$ to the region near $\lambda^{\alpha}=0$ and eliminates the divergence coming from functional integration over the non-compact zero modes of $\lambda^{\alpha}$.

To quantize this action, one needs to gauge-fix the invariances generated by the constraint $C_{\alpha}$ of (1.3). But because $\lambda \gamma^{m} \lambda=0$, only 5 of the 16 components of $C_{\alpha}$ are independent. To gauge-fix, one should first restrict $\lambda^{\alpha}$ to a patch of pure spinor space where $\bar{\lambda}_{\alpha} \lambda^{\alpha} \neq 0$ for some fixed constant pure spinor $\bar{\lambda}_{\alpha}$. On this patch of pure spinor space, one can restrict the Lagrange multiplier $L^{\alpha}$ to satisfy the 11 independent constraints $L \gamma^{m n} \bar{\lambda}=0$, and the remaining 5 components of $L^{\alpha}$ can be gauge-fixed in the usual manner to produce 5 fermionic Faddeev-Popov ghosts and antighosts, $f^{\alpha}$ and $m_{\alpha}$, which satisfy the constraints $f \gamma^{m n} \bar{\lambda}=0$ and $m \gamma^{m n} \lambda=0$.

On this same patch of pure spinor space, one can similarly gauge-fix $\bar{\Lambda}_{\alpha}$ to be proportional to the constant pure spinor $\bar{\lambda}_{\alpha}$. This gauge-fixing procedure produces additional fermionic Faddeev-Popov ghosts and antighosts, $g_{\alpha}$ and $n^{\alpha}$, which because of the pure spinor constraint on $\bar{\Lambda}_{\alpha}$, are constrained to satisfy $g \gamma^{m} \bar{\lambda}=0$ and $n \gamma^{m} \lambda=0$ so they each have 11 independent components.

Note that there are no additional Faddeev-Popov ghosts and antighosts coming from gauge-fixing worldsheet reparameterization invariance to conformal gauge since the Virasoro constaint $T=0$ is already implied by the twistor-like constraint of (1.3). This explains why the $b$ ghost satisfying $\{Q, b\}=T$ is not a fundamental worldsheet variable in the pure spinor formalism, but is a composite operator constructed out of the other variables.

Although the constraints on the fermionic ghosts $\left(f^{\alpha}, g_{\alpha}\right)$ and antighosts $\left(m_{\alpha}, n^{\alpha}\right)$ depend on the choice of patch of pure spinor space, one can define an unconstrained fermionic spinor variable $\theta^{\alpha}$ and its conjugate momentum $p_{\alpha}$ as

$$
\theta^{\alpha}=f^{\alpha}+n^{\alpha} \quad \text { and } \quad p_{\alpha}=g_{\alpha}+m_{\alpha}
$$

which are independent of the choice of $\bar{\lambda}_{\alpha}$. In terms of these unconstrained fermionic variables, the Faddeev-Popov ghost contribution to the action is

$$
\int d^{2} z\left(m_{\alpha} \bar{\partial} f^{\alpha}+n^{\alpha} \bar{\partial} g_{\alpha}\right)=\int d^{2} z p_{\alpha} \bar{\partial} \theta^{\alpha}
$$

so the gauge-fixed action is

$$
S=\int d^{2} z\left(\frac{1}{2} \partial x^{m} \bar{\partial} x_{m}+w_{\alpha} \bar{\partial} \lambda^{\alpha}+p_{\alpha} \bar{\partial} \theta^{\alpha}\right) .
$$


And the resulting BRST operator is

$$
Q=\int d z\left(\lambda^{\alpha} p_{\alpha}+C_{\alpha} \theta^{\alpha}-\frac{1}{8}\left(\lambda \gamma^{m} \theta\right)\left(\theta \gamma_{m} \partial \theta\right)\right)=\int d z \lambda^{\alpha} d_{\alpha}
$$

where $d_{\alpha}$ is the supersymmetric Green-Schwarz-Siegel constraint [14] and the term $-\frac{1}{8}\left(\lambda \gamma^{m} \theta\right)\left(\theta \gamma_{m} \partial \theta\right)$ in $Q$ comes from the non-abelian constraint algebra $\left[C_{\alpha}, C_{\beta}\right]=$ $\frac{1}{8}\left(\gamma^{m} \lambda\right)_{[\alpha}\left(\gamma_{m} \nabla \lambda\right)_{\beta]}$.

So the gauge-fixed action and BRST operator of the pure spinor formalism are obtained by quantizing the simple worldsheet reparameterization invariant action of (1.4). Since the fermionic worldsheet variables in the gauge-fixed action come from FaddeevPopov ghosts and antighosts, a natural question is how fermionic variables can appear in light-cone gauge where ghosts are absent. This question can be studied by simplifying to the $\mathrm{d}=10$ superparticle [42] where the twistor-like constraint of (1.3) reduces to $C_{\alpha}=-\frac{1}{2} P^{m}\left(\gamma_{m} \lambda\right)_{\alpha}=0$. Although $C_{\alpha}$ has 5 independent components, it implies a single mass-shell constraint $P^{2}=0$ for the $x^{m}$ dependence. And since $\lambda^{\alpha}$ dependence is fixed by the $\lambda^{\alpha} \bar{\Lambda}_{\alpha}$ term in the Lagrangian to be near $\lambda^{\alpha}=0$, there are 4 components of $C_{\alpha}$ which overconstrain the classical worldsheet variables. These 4 extra constraints of $C_{\alpha}$ lead to 4 fermionic variables together with their conjugate momenta which are the usual 8 light-cone Green-Schwarz fermions.

It is interesting to point out that this same phenomenon occurs for the $d=11$ pure spinor description of the superparticle [43-46] which describes $d=11$ supergravity. In this case, the bosonic variables are $x^{M}$ for $M=0$ to 10 and $\lambda^{A}$ for $A=1$ to 32 where $\lambda^{A}$ satisfies the pure spinor constraint $\lambda \gamma^{M} \lambda=0$ that reduces its 32 components to 23 independent components. The twistor-like constraint $C_{A}=-\frac{1}{2} P^{M}\left(\gamma_{M} \lambda\right)_{A}=0$ has 9 independent components, and implies the $\mathrm{d}=11$ mass-shell constraint $P^{2}=0$. So there are 8 components of $C_{A}$ which overconstrain the classical variables and lead to 8 fermionic variables and their conjugate momenta in light-cone gauge.

After describing the worldsheet reparameterization invariant action of (1.4) and its gauge invariances in sections 2 and 3 of this paper, the gauge-fixing procedure on a patch where $\bar{\lambda}_{\alpha} \lambda^{\alpha} \neq 0$ will be discussed in section 4 . In section 5 , the "minimal" version of the pure spinor formalism will be derived using this procedure, and in section 6 , the "nonminimal" version of the pure spinor formalism $[47,48]$ will be derived by upgrading $\bar{\lambda}_{\alpha}$ from a constant pure spinor to a worldsheet variable.

Finally, a conjecture for generalizing this procedure to curved Type II supergravity backgrounds including Ramond-Ramond fields will be proposed in section 7 . Since all fermionic variables in the worldsheet action arise from Faddeev-Popov ghosts, RamondRamond background fields will not directly appear in the reparameterization invariant action and will only appear after performing the gauge-fixing procedure. The absence of physical Ramond-Ramond fields from the classical action implies that there is non-trivial BRST cohomology at nonzero ghost number where the ghosts $\left(f^{\alpha}, g_{\alpha}\right)$ and antighosts $\left(m_{\alpha}, n^{\alpha}\right)$ are defined to carry ghost-number +1 and -1 . This fact is not surprising since the patch-independent variables $\theta^{\alpha}$ and $p_{\alpha}$ of (1.5) do not have well-defined ghost number when ghost number is defined in terms of $\left(f^{\alpha}, g_{\alpha}\right)$ and $\left(m_{\alpha}, n^{\alpha}\right)$. 


\section{Worldsheet action}

The worldsheet variables in the reparameterization invariant action will include the spacetime $x^{m}$ variables $(m=0$ to 9$)$, the left-moving bosonic pure spinor $\lambda^{\alpha}$ variables $(\alpha=1$ to 16) and their conjugate momenta $w_{\alpha}$, and the right-moving bosonic pure spinor $\hat{\lambda}^{\hat{\alpha}}$ variables and their conjugate momenta $\widehat{w}_{\hat{\alpha}}$. Because of the pure spinor constraints

$$
\lambda \gamma^{m} \lambda=\widehat{\lambda} \gamma^{m} \widehat{\lambda}=0,
$$

the conjugate momenta $w_{\alpha}$ and $\widehat{w}_{\hat{\alpha}}$ can only appear in combinations which are invariant under the gauge transformations $\delta w_{\alpha}=c^{m}\left(\gamma_{m} \lambda\right)_{\alpha}$ and $\delta \widehat{w}_{\hat{\alpha}}=\widehat{c}^{m}\left(\gamma_{m} \widehat{\lambda}\right)_{\hat{\alpha}}$ for arbitrary $c^{m}$ and $\widehat{c}^{m}$. Note that $\left(x^{m}, \lambda^{\alpha}, \widehat{\lambda}^{\hat{\alpha}}\right)$ are worldsheet scalars, and $w_{\alpha}$ and $\widehat{w}_{\hat{\alpha}}$ carry conformal weight $(1,0)$ and $(0,1)$ respectively. For the Type IIA (or Type IIB) superstring, the $\hat{\alpha}$ index on right-moving spinors denotes the opposite (or same) spacetime chirality as the unhatted $\alpha$ index on left-moving spinors. And the heterotic superstring is obtained by replacing the right-moving sector with the same right-moving sector as in the RNS heterotic formalism.

The Type II worldsheet action in a flat background is

$$
\begin{aligned}
S=\int d^{2} z(\operatorname{det} e)\left[\frac{1}{2} \nabla\right. & x^{m} \bar{\nabla} x_{m}+w_{\alpha} \bar{\nabla} \lambda^{\alpha}+\widehat{w}_{\hat{\alpha}} \nabla \widehat{\lambda}^{\hat{\alpha}} \\
& \left.\quad+L^{\alpha} C_{\alpha}+\bar{\Lambda}_{\alpha} \lambda^{\alpha}+\widehat{L}^{\hat{\alpha}} \widehat{C}_{\hat{\alpha}}+\widehat{\bar{\Lambda}}_{\hat{\alpha}} \widehat{\lambda}^{\hat{\alpha}}+\frac{1}{4}\left(L \gamma^{m} \lambda\right)\left(\widehat{L} \gamma_{m} \widehat{\lambda}\right)\right]
\end{aligned}
$$

where $\nabla=e_{-}^{J} \partial_{J}, \bar{\nabla}=e_{+}^{J} \partial_{J}, e_{ \pm}^{J}$ is the worldsheet vielbein for $J=1$ to $2, C_{\alpha}$ and $\widehat{C}_{\hat{\alpha}}$ are the twistor-like constraints

$$
C_{\alpha}=-\frac{1}{2} \nabla x^{m}\left(\gamma_{m} \lambda\right)_{\alpha}, \quad \widehat{C}_{\hat{\alpha}}=-\frac{1}{2} \bar{\nabla} x^{m}\left(\gamma_{m} \widehat{\lambda}\right)_{\hat{\alpha}},
$$

$L^{\alpha}$ and $\widehat{L}^{\hat{\alpha}}$ are Lagrange multipliers of conformal weight $(0,1)$ and $(1,0)$, and $\bar{\Lambda}_{\alpha}$ and $\widehat{\bar{\Lambda}}_{\hat{\alpha}}$ are Lagrange multipliers of conformal weight $(1,1)$.

Just as $\lambda^{\alpha}$ and $\widehat{\lambda}^{\hat{\alpha}}$ are pure spinors satisfying the constraint of (2.1), the Lagrange multipliers $\bar{\Lambda}_{\alpha}$ and $\widehat{\bar{\Lambda}}_{\hat{\alpha}}$ will also be required to be pure spinors satisfying the constraints

$$
\bar{\Lambda} \gamma^{m} \bar{\Lambda}=\widehat{\bar{\Lambda}} \gamma^{m} \widehat{\bar{\Lambda}}=0
$$

so that $\bar{\Lambda}_{\alpha}$ and $\widehat{\bar{\Lambda}}_{\hat{\alpha}}$ each have 11 independent complex components. After Wick rotation to Euclidean signature, pure spinors parameterize the complex space $\frac{\mathrm{SO}(10)}{\mathrm{U}(5)} \times C^{*}$ where $C^{*}$ denotes the complex plane minus the origin. So all components of a pure spinor cannot be simultaneously zero. To globally paramaterize pure spinors, one therefore needs to divide the space into 16 patches $\mathcal{O}_{\alpha}$ for $\alpha=1$ to 16 where, on the patch $\mathcal{O}_{\alpha}$, the component $\lambda^{\alpha}$ and $\bar{\Lambda}_{\alpha}$ of the pure spinors are required to be nonvanishing [19].

In addition to acting as a Lagrange multiplier for the nonzero modes of $\lambda^{\alpha}$, the zero modes of $\bar{\Lambda}_{\alpha}$ can be interpreted as a regulator for the zero modes of $\lambda^{\alpha}$. In other words, if the zero modes of $\bar{\Lambda}_{\alpha}$ are interpreted (after Wick rotation) as the complex conjugate 
of the $\lambda^{\alpha}$ zero modes, the term $\lambda^{\alpha} \bar{\Lambda}_{\alpha}$ in the action acts as a Gaussian regulator for the functional integration over these non-compact pure spinor zero modes. Note that the pure spinor constraint on $\bar{\Lambda}_{\alpha}$ implies that it cannot be used to remove all $\lambda^{\alpha}$ dependence from the action of (2.2). For example, the shift

$$
\bar{\Lambda}_{\alpha} \rightarrow \bar{\Lambda}_{\alpha}+\bar{\nabla} w_{\alpha}+\frac{1}{2} \nabla x^{m}\left(\gamma_{m} L\right)_{\alpha}
$$

which would naively remove $\lambda^{\alpha}$ dependence from the action is not allowed since it does not preserve the constraint of (2.4).

To simplify notation, the right-moving sector will be ignored for the rest of this paper when it plays an identical role to the left-moving sector.

\section{Gauge invariances}

Because of the first-class constraint $C_{\alpha}=-\frac{1}{2} \nabla x^{m}\left(\gamma_{m} \lambda\right)_{\alpha}$, the worldsheet action of (2.2) is invariant under the gauge transformation

$$
\begin{aligned}
\delta x^{m} & =\frac{1}{2} \lambda \gamma^{m} f, \quad \delta w_{\alpha}=-\frac{1}{2} \nabla x^{m}\left(\gamma_{m} f\right)_{\alpha}+\frac{1}{4}\left(\widehat{L} \gamma^{m} \widehat{\lambda}\right)\left(\gamma_{m} f\right)_{\alpha}, \quad \delta L^{\alpha}=\bar{\nabla} f^{\alpha}, \\
\delta \bar{\Lambda}_{\alpha} & =\frac{1}{16(\lambda \bar{\Lambda})}\left(\gamma^{m} \gamma^{n} \bar{\Lambda}\right)_{\alpha}\left[\nabla\left(\lambda \gamma_{m} f\right)\left(\lambda \gamma_{n} L\right)-\nabla\left(\lambda \gamma_{m} L\right)\left(\lambda \gamma_{n} f\right)\right]
\end{aligned}
$$

where $f^{\alpha}$ is an arbitrary infinitesimal parameter and the variation of $\delta \bar{\Lambda}_{\alpha}$ is necessary since $\left[C_{\alpha}, C_{\beta}\right]=\frac{1}{8}\left(\gamma_{m} \lambda\right)_{[\alpha}\left(\gamma_{n} \nabla \lambda\right)_{\beta]}$ implies that

$$
\lambda^{\alpha} \delta \bar{\Lambda}_{\alpha}=\frac{1}{8}\left[\left(\lambda \gamma^{m} L\right) \nabla\left(\lambda \gamma_{m} f\right)-\left(\lambda \gamma^{m} f\right) \nabla\left(\lambda \gamma_{m} L\right)\right] .
$$

Although (3.3) does not uniquely determine (3.2), it will be later argued that any other $\delta \bar{\Lambda}_{\alpha}$ that satisfies (3.3) will lead to the same BRST operator up to a similarity transformation.

The gauge invariance $x^{m} \sim x^{m}+\frac{1}{2} \lambda \gamma^{m} f$ of (3.1) is the $\mathrm{d}=10$ generalization of the $\mathrm{d}=4$ twistor symmetry [30]

$$
x^{a \dot{a}} \sim x^{a \dot{a}}+\lambda^{a} f^{\dot{a}} \quad \text { where } a, \dot{a}=1 \text { to } 2
$$

that identifies points on a self-dual plane and leaves the twistor variable $\mu^{\dot{a}}=x^{a \dot{a}} \lambda_{a}$ invariant. So as discussed in [20-29], the $\mathrm{d}=10$ pure spinor variable $\lambda^{\alpha}$ plays a similar role to the $\mathrm{d}=4$ twistor variable $\lambda^{a}$ of Penrose.

The worldsheet action of (2.2) is also invariant under the gauge transformation generated by $\lambda^{\alpha}$ which is

$$
\delta w_{\alpha}=g_{\alpha}, \quad \delta \bar{\Lambda}_{\alpha}=\bar{\nabla} g_{\alpha}+\frac{1}{2(\lambda \bar{\Lambda})}\left(g \gamma^{m} \overline{\nabla \Lambda}\right)\left(\gamma_{m} \lambda\right)_{\alpha},
$$

where $g_{\alpha}$ is an arbitrary infinitesimal parameter of conformal weight $(1,0)$ satisfying $\left(g \gamma^{m} \bar{\Lambda}\right)=0$ and the second term in $\delta \bar{\Lambda}_{\alpha}$ is needed so that $\delta \bar{\Lambda} \gamma^{m} \bar{\Lambda}=0$. Furthermore, since $\lambda \gamma^{m} \lambda=0,(2.2)$ is invariant under the gauge transformations

$$
\delta L^{\alpha}=c_{m n}\left(\gamma^{m n} \lambda\right)^{\alpha}
$$


for arbitrary $c^{m n}$, which implies that 11 of the 16 components of $L^{\alpha}$ can be gauged away.

Finally, the worldsheet action is invariant under the usual worldsheet reparameterizations generated by the Virasoro constraint

$$
T=-\frac{1}{2} \nabla x^{m} \nabla x_{m}-w_{\alpha} \nabla \lambda^{\alpha} .
$$

However, these reparameterizations are already included as a special case of the previous gauge transformations. This can be seen from the fact that the Virasoro constraint of (3.7) can be expressed as a linear combination of the other constraints $C_{\alpha}=-\frac{1}{2} \nabla x^{m}\left(\gamma_{m} \lambda\right)_{\alpha}$ and $\lambda^{\alpha}$ as

$$
T=C_{\alpha} \frac{\nabla x^{m}\left(\gamma_{m} \bar{\Lambda}\right)^{\alpha}}{(\lambda \bar{\Lambda})}+\nabla \lambda^{\alpha} \frac{\left(\lambda \gamma_{m n} w\right)\left(\gamma^{m n} \bar{\Lambda}\right)_{\alpha}+2(\lambda w) \bar{\Lambda}_{\alpha}}{8(\lambda \bar{\Lambda})} .
$$

So all dependence of the action of (2.2) on off-diagonal components of the worldsheet vierbein can be removed by an appropriate shift of the Lagrange multipliers $\left(L^{\alpha}, \bar{\Lambda}_{\alpha}\right)$ and $\left(\widehat{L}^{\hat{\alpha}}, \widehat{\bar{\Lambda}}_{\hat{\alpha}}\right)$.

\section{Gauge fixing}

After shifting the Lagrange multipliers to eliminate the off-diagonal components of the worldsheet vierbien, the worldsheet action can be expressed in conformal gauge where $e_{ \pm}^{J}$ is proportional to $\delta_{ \pm}^{J}$ so that $\nabla \rightarrow \partial$ and $\bar{\nabla} \rightarrow \bar{\partial}$. One then needs to fix the gauge invariances of (3.1), (3.5) and (3.6). The first step to perform this gauge fixing is to restrict the pure spinor $\lambda^{\alpha}$ to a patch $\mathcal{O}_{\alpha}$ where one of its components is required to be nonzero. This patch can be defined by introducing a constant pure spinor $\bar{\lambda}_{\alpha}$ and requiring that $\bar{\lambda}_{\alpha} \lambda^{\alpha}$ is nonzero on the patch. Different choices of the constant pure spinor $\bar{\lambda}_{\alpha}$ correspond to different patches $\mathcal{O}_{\alpha}$, and consistency of the gauge fixing will require that the resulting gauge-fixed action and BRST operator are independent of the choice of $\bar{\lambda}_{\alpha}$.

On the patch where $\bar{\lambda}_{\alpha} \lambda^{\alpha}$ is nonzero, the gauge invariance of (3.6) implies that one can gauge fix $L \gamma^{m n} \bar{\lambda}=0$, which fixes 11 of the 16 components of $L^{\alpha}$. The remaining 5 components of $L^{\alpha}$ will be gauge-fixed to zero using the invariance of (3.1) in which the gauge parameter $f^{\alpha}$ is also constrained to satisfy

$$
f \gamma^{m n} \bar{\lambda}=0
$$

Finally, the gauge parameter $g_{\alpha}$ of (3.5) can be used to gauge-fix the Lagrange multipler $\bar{\Lambda}_{\alpha}$ to satisfy

$$
\bar{\Lambda}_{\alpha}=\epsilon \bar{\lambda}_{\alpha}
$$

where $\epsilon$ is a constant. Note that $\bar{\Lambda}_{\alpha}$ cannot be gauge-fixed to zero since it is a pure spinor taking values in $\frac{\mathrm{SO}(10)}{\mathrm{U}(5)} \times C^{*}$. In the gauge of (4.2), $g_{\alpha}$ satisfies the constraint $g \gamma^{m} \bar{\lambda}=0$.

One can now follow the standard BRST procedure where the gauge parameters $f^{\alpha}$ and $g_{\alpha}$ are interpreted as fermionic ghosts, and fermionic antighosts $m_{\alpha}$ and $n^{\alpha}$ are introduced due to the gauge-fixing of the Lagrange multipliers $L^{\alpha}$ and $\bar{\Lambda}_{\alpha}$. But because the Virasoro constraint $T$ can be expressed in terms of $C_{\alpha}$ and $\lambda^{\alpha}$ as in (3.8), there is no 
need to introduce the usual Virasoro ghost and antighost, $c$ and $b$, from gauge-fixing the reparameterization invariance. ${ }^{1}$

The resulting gauge-fixed action is

$$
\begin{aligned}
S=S_{0}-\int d^{2} z Q & \left(m_{\alpha} L^{\alpha}+n^{\alpha}\left(\bar{\Lambda}_{\alpha}-\epsilon \bar{\lambda}_{\alpha}\right)\right)-\int d^{2} z \widehat{Q}\left(\widehat{m}_{\hat{\alpha}} \widehat{L}^{\hat{\alpha}}+\widehat{n}^{\hat{\alpha}}\left(\widehat{\bar{\Lambda}}_{\hat{\alpha}}-\widehat{\epsilon} \widehat{\bar{\lambda}}_{\hat{\alpha}}\right)\right) \\
=\int d^{2} z \frac{1}{2} \partial x m & \bar{\partial} x_{m}+w_{\alpha} \bar{\partial} \lambda^{\alpha}+\widehat{w}_{\hat{\alpha}} \partial \widehat{\lambda}^{\hat{\alpha}} \\
& +L^{\alpha} C_{\alpha}+\bar{\Lambda}_{\alpha} \lambda^{\alpha}+\widehat{L}^{\hat{\alpha}} \widehat{C}_{\hat{\alpha}}+\widehat{\bar{\Lambda}}_{\hat{\alpha}} \widehat{\lambda}^{\hat{\alpha}}+\frac{1}{4}\left(L \gamma^{m} \lambda\right)\left(\widehat{L} \gamma_{m} \widehat{\lambda}\right) \\
& -M_{\alpha} L^{\alpha}-N^{\alpha}\left(\bar{\Lambda}_{\alpha}-\epsilon \bar{\lambda}_{\alpha}\right)+m_{\alpha} \bar{\partial} f^{\alpha} \\
& +n^{\alpha}\left(\bar{\partial} g_{\alpha}+\frac{1}{8}\left(\gamma^{m} L\right)_{\alpha} \partial\left(\lambda \gamma_{m} f\right)-\frac{1}{8}\left(\gamma^{m} f\right) \partial\left(\lambda \gamma^{m} L\right)\right) \\
& -\widehat{M}_{\hat{\alpha}} \widehat{L}^{\hat{\alpha}}-\widehat{N}^{\hat{\alpha}}\left(\widehat{\bar{\Lambda}}_{\hat{\alpha}}-\widehat{\epsilon} \widehat{\bar{\lambda}}_{\hat{\alpha}}\right)+\widehat{m}_{\hat{\alpha}} \partial \widehat{f}^{\hat{\alpha}} \\
& \left.+\widehat{n}^{\hat{\alpha}}\left(\partial \widehat{g}_{\hat{\alpha}}+\frac{1}{8}\left(\gamma^{m} \widehat{L}\right)_{\hat{\alpha}} \bar{\partial}\left(\widehat{\lambda} \gamma_{m} \widehat{f}\right)-\frac{1}{8}\left(\gamma^{m} \widehat{f}\right) \bar{\partial}\left(\widehat{\lambda} \gamma^{m} \widehat{L}\right)\right)\right]
\end{aligned}
$$

where $S_{0}$ is the action of (2.2) in conformal gauge, $\left(M_{\alpha}, N^{\alpha}\right)$ are bosonic Nakanishi-Lautrup fields associated with the gauge-fixing of $L^{\alpha}$ and $\bar{\Lambda}_{\alpha}$, and

$$
Q=\int d z\left[\lambda^{\alpha} g_{\alpha}+C_{\alpha} f^{\alpha}-\frac{1}{8}\left(n \gamma^{m} f\right) \partial\left(\lambda \gamma_{m} f\right)\right]
$$

is the BRST operator which generates the BRST transformations

$$
\begin{aligned}
Q x^{m} & =\frac{1}{2} \lambda \gamma^{m} f, \quad Q w_{\alpha}=g_{\alpha}+\ldots, \quad Q L^{\alpha}=\bar{\partial} f^{\alpha}, \quad Q f^{\alpha}=0, \\
Q g_{\alpha} & =-\frac{1}{8}\left(\gamma^{m} f\right)_{\alpha} \partial\left(\lambda \gamma_{m} f\right), \quad Q m_{\alpha}=M_{\alpha}, \quad Q n^{\alpha}=N^{\alpha}, \\
Q \bar{\Lambda}_{\alpha} & =\bar{\partial} g_{\alpha}+\frac{1}{16(\lambda \bar{\Lambda})}\left(\gamma^{m} \gamma^{n} \bar{\Lambda}\right)_{\alpha}\left[\partial\left(\lambda \gamma_{m} f\right)\left(\lambda \gamma_{n} L\right)-\partial\left(\lambda \gamma_{m} L\right)\left(\lambda \gamma_{n} f\right)\right] .
\end{aligned}
$$

\footnotetext{
${ }^{1}$ If desired, one can treat the invariances generated by $T$ as independent symmetries if one also includes the gauge-for-gauge invariances implied by the relation of (3.8). In this case, the gauge-fixing procedure will generate the usual fermionic $(b, c)$ Virasoro ghosts of conformal wieght $(2,-1)$ together with a set of bosonic ghost-for-ghosts $(\beta, \gamma)$ which also carry conformal weight $(2,-1)$. Although it will not be verified here, it is expected that these ghosts and ghost-for-ghosts will contribute to the BRST operator the terms

$$
Q=Q_{0}+\int d z[\gamma(b-B)+c(T-b \partial c-\beta \partial \gamma-\partial(\beta \gamma)]
$$

where $Q_{0}=\int d z\left(\lambda^{a} d_{\alpha}+\bar{w}^{\alpha} r_{\alpha}\right)$ is the usual non-minimal pure spinor BRST operator and

$$
B=d_{\alpha} \frac{\left(\partial x^{m}+\frac{1}{2} \theta \gamma^{m} \partial \theta\right)\left(\gamma_{m} \bar{\lambda}\right)^{\alpha}}{(\lambda \bar{\lambda})}+\partial \theta^{\alpha} \frac{\left(\lambda \gamma_{m n} w\right)\left(\gamma^{m n} \bar{\lambda}\right)_{\alpha}+2(\lambda w) \bar{\lambda}_{\alpha}}{8(\lambda \bar{\lambda})}+\ldots
$$

is the composite ghost satisfying $\left\{Q_{0}, B\right\}=T$ with $\ldots$ denoting terms depending on the non-minimal variables $\left(r_{\alpha}, s^{\alpha}\right)$. Note that $Q=e^{U}\left(Q_{0}+\gamma b\right) e^{-U}$ where $U=\int d z(c B-c \partial c \beta)$ and that the structure of $B$ in (4.4) resembles the structure of (3.8).
} 
Since $L \gamma^{m n} \bar{\lambda}=0$ and $\bar{\Lambda} \gamma^{m} \bar{\Lambda}=0$ imply that only 5 components of $L^{\alpha}$ and 11 components of $\bar{\Lambda}_{\alpha}$ are independent, one can choose the antighosts and Nakanashi-Lautrup fields to satisfy the constraints

$$
\lambda \gamma^{m n} m=\lambda \gamma^{m n} M=0 \quad \text { and } \quad \lambda \gamma^{m} n=\lambda \gamma^{m} N=0 .
$$

\section{Gauge-fixed pure spinor formalism}

After integrating out the Lagrange multipliers and Nakanashi-Lautrup fields, one obtains the equations

$$
\begin{aligned}
L^{\alpha} & =0, & \bar{\Lambda}_{\alpha}-\epsilon \bar{\lambda}_{\alpha} & =0 \\
M_{\alpha} & =C_{\alpha}+\frac{1}{8}\left(\gamma^{m} n\right)_{\alpha} \partial\left(\lambda \gamma_{m} f\right)+\frac{1}{8}\left(\gamma^{m} \lambda\right)_{\alpha} \partial\left(n \gamma_{m} f\right), & N^{\alpha} & =\lambda^{\alpha}
\end{aligned}
$$

and the action

$$
S=\int d^{2} z\left(\frac{1}{2} \partial x^{m} \bar{\partial} x_{m}+w_{\alpha} \bar{\partial} \lambda^{\alpha}+\widehat{w}_{\hat{\alpha}} \partial \widehat{\lambda}^{\hat{\alpha}}+m_{\alpha} \bar{\partial} f^{\alpha}+n^{\alpha} \bar{\partial} g_{\alpha}+\widehat{m}_{\hat{\alpha}} \partial \widehat{f}^{\hat{\alpha}}+\widehat{n}^{\hat{\alpha}} \partial \widehat{g}_{\hat{\alpha}}\right)
$$

Since $\bar{\lambda}_{\alpha}$ appears in the action of (5.2) and in the BRST operator of (4.6) through the constraints on the ghosts and antighosts, this gauge fixing naively appears to depend on the choice of patch $\mathcal{O}_{\alpha}$. However, after a cleverly chosen field redefinition, all dependence on $\bar{\lambda}_{\alpha}$ can be eliminated from the action and the BRST operator, and one can take the limit $\epsilon \rightarrow 0$ in the gauge-fixing condition $\bar{\Lambda}_{\alpha}=\epsilon \bar{\lambda}_{\alpha}$.

The field redefinition involves defining a new unconstrained fermionic variable $\theta^{\alpha}$ and its conjugate momentum $p_{\alpha}$ in terms of the constrained variables $\left(f^{\alpha}, g_{\alpha}, m_{\alpha}, n^{\alpha}\right)$ as

$$
\theta^{\alpha}=f^{\alpha}+n^{\alpha} \text { and } p_{\alpha}=e^{R}\left(g_{\alpha}+m_{\alpha}\right) e^{-R}
$$

where

$$
R=-\frac{1}{24} \int d z\left[\left(n \gamma^{m} \partial n\right)\left(n \gamma_{m} f\right)+3\left(n \gamma^{m} \partial f\right)\left(n \gamma_{m} f\right)\right]
$$

Note that

$$
\lambda^{\alpha} p_{\alpha}=e^{R}\left(\lambda^{\alpha} g_{\alpha}\right) e^{-R}=\lambda^{\alpha} g_{\alpha}+\frac{1}{8}\left(\lambda \gamma^{m} f\right)\left(n \gamma_{m} \partial n\right)+\frac{1}{4}\left(\lambda \gamma^{m} f\right)\left(n \gamma_{m} \partial f\right),
$$

and if one had chosen a different $\delta \bar{\Lambda}_{\alpha}$ in (3.2) which also satisfied (3.3), the similarity transformation $R$ of (5.4) would be modified in a manner to leave the BRST operator invariant when expressed in terms of $\theta^{\alpha}$ and $p_{\alpha}$.

It is easy to verify that all 16 components of $\theta^{\alpha}$ and $p_{\alpha}$ in (5.3) are unconstrained since the 5 independent components of $f^{\alpha}$ and $m_{\alpha}$ are in different directions from the 11 independent components of $g_{\alpha}$ and $n^{\alpha}$. However, since $\left(f^{\alpha}, g_{\alpha}\right)$ and $\left(m_{\alpha}, n^{\alpha}\right)$ are ghosts and antighosts which carry conventional ghost number +1 and $-1, \theta^{\alpha}$ and $p_{\alpha}$ of (5.3) do not have well-defined ghost number with respect to the conventional definition. Nevertheless, one can define a new ghost number where $\left(x^{m}, \theta^{\alpha}, p_{\alpha}\right)$ carry zero ghost number and $\left(\lambda^{\alpha}, w_{\alpha}\right)$ carry ghost number $(+1,-1)$. With respect to this new ghost number, the worldsheet 
action will carry zero ghost number and the BRST operator will carry +1 ghost number as desired.

After a suitable shift of $w_{\alpha}$ to absorb terms proportional to $\bar{\partial} \lambda^{\alpha}$, the action and BRST operator of (5.2) and (4.6) can be simply expressed in terms of $\theta^{\alpha}$ and $p_{\alpha}$ of (5.3) as

$$
\begin{aligned}
S & =\int d^{2} z\left(\frac{1}{2} \partial x^{m} \bar{\partial} x_{m}+w_{\alpha} \bar{\partial} \lambda^{\alpha}+\widehat{w}_{\hat{\alpha}} \partial \widehat{\lambda}^{\hat{\alpha}}+p_{\alpha} \bar{\partial} \theta^{\alpha}+\widehat{p}_{\hat{\alpha}} \partial \widehat{\theta}^{\hat{\alpha}}\right), \\
Q & =\int d z\left(\lambda^{\alpha} p_{\alpha}-\frac{1}{2} \partial x^{m}\left(\lambda \gamma_{m} \theta\right)-\frac{1}{8}\left(\lambda \gamma^{m} \theta\right)\left(\theta \gamma_{m} \partial \theta\right)\right)=\int d z \lambda^{\alpha} d_{\alpha}
\end{aligned}
$$

where

$$
d_{\alpha}=p_{\alpha}-\frac{1}{2} \partial x^{m}\left(\gamma_{m} \theta\right)_{\alpha}-\frac{1}{8}\left(\gamma^{m} \theta\right)_{\alpha}\left(\theta \gamma_{m} \partial \theta\right)
$$

is the spacetime supersymmetric Green-Schwarz-Siegel constraint. So one recovers the spacetime supersymmetric gauge-fixed action and BRST operator of the "minimal" pure spinor formalism which is manifestly independent of the choce of $\bar{\lambda}_{\alpha}$.

\section{Gauge-fixed non-minimal pure spinor formalism}

To obtain the non-minimal pure spinor formalism [47, 48] from gauge fixing, one upgrades the constant pure spinor $\bar{\lambda}_{\alpha}$ to a worldsheet variable and constrains its conjugate momentum $\bar{w}^{\alpha}$ to vanish by adding the term

$$
\int d^{2} z\left(\bar{w}^{\alpha} \bar{\nabla}_{\alpha}+\bar{w}^{\alpha} H_{\alpha}\right)
$$

to the action of (5.6) where $H_{\alpha}$ is a Lagrange multiplier for the constraint $\bar{w}^{\alpha}=0$. Since only 11 components of $\bar{w}^{\alpha}$ are independent, the Lagrange multiplier needs to be constrained to satisfy $H \gamma^{m} \bar{\lambda}=0$.

When expressed in terms of $\theta^{\alpha}$ and $p_{\alpha}$, the action of (5.6) is independent of $\bar{\lambda}_{\alpha}$ in the limit where the constant $\epsilon$ of $\bar{\Lambda}_{\alpha}=\epsilon \bar{\lambda}_{\alpha}$ is taken to zero. To obtain the gauge-fixed nonminimal formalism, one leaves $\epsilon$ nonzero and defines the non-minimal contribution to the BRST transformations of (4.7) as

$$
Q \bar{\lambda}_{\alpha}=-r_{\alpha}, \quad Q H_{\alpha}=\bar{\nabla} r_{\alpha}, \quad Q s^{\alpha}=S^{\alpha},
$$

where $r_{\alpha}$ is the fermionic ghost constrained to satisfy $r \gamma^{m} \bar{\lambda}=0$, and $s^{\alpha}$ and $S^{\alpha}$ are the antighost and Nakanishi-Lautrup field associated to $H_{\alpha}$. Note that since $\theta^{\alpha}$ and $p_{\alpha}$ are defined to be independent of $\bar{\lambda}_{\alpha}$, their BRST transformations do not involve $r_{\alpha}$ and are

$$
Q \theta^{\alpha}=\lambda^{\alpha}, \quad Q p_{\alpha}=C_{\alpha}-\frac{1}{8}\left[\left(\theta \gamma^{m} \partial \theta\right)\left(\gamma_{m} \lambda\right)_{\alpha}-\partial\left(\lambda \gamma^{m} \theta\right)\left(\gamma_{m} \theta\right)_{\alpha}-2\left(\lambda \gamma^{m} \theta\right)\left(\gamma_{m} \partial \theta\right)_{\alpha}\right]
$$

After gauge-fixing $H_{\alpha}=0$, the resulting gauge-fixed action and BRST operator are

$$
\begin{aligned}
S & =\int d^{2} z\left[\frac{1}{2} \partial x^{m} \bar{\partial} x_{m}+w_{\alpha} \bar{\partial} \lambda^{\alpha}+\widehat{w}_{\hat{\alpha}} \partial \widehat{\lambda}^{\hat{\alpha}}+p_{\alpha} \bar{\partial} \theta^{\alpha}+\widehat{p}_{\hat{\alpha}} \partial \widehat{\theta}^{\hat{\alpha}}\right. \\
& \left.+\bar{w}^{\alpha} \bar{\partial} \lambda_{\alpha}+\widehat{\bar{w}}^{\hat{\alpha}} \partial \widehat{\lambda}_{\hat{\alpha}}+s^{\alpha} \bar{\partial} r_{\alpha}+\widehat{s}^{\hat{\alpha}} \partial \widehat{r}_{\hat{\alpha}}+\epsilon\left(\lambda^{\alpha} \bar{\lambda}_{\alpha}+\theta^{\alpha} r_{\alpha}\right)+\widehat{\epsilon}\left(\widehat{\lambda}^{\hat{\alpha}} \widehat{\bar{\lambda}}_{\hat{\alpha}}+\widehat{\theta}^{\hat{\alpha}} \widehat{r}_{\hat{\alpha}}\right)\right], \\
Q & =\int d z\left(\lambda^{\alpha} d_{\alpha}+\bar{w}^{\alpha} r_{\alpha}\right),
\end{aligned}
$$


where the term $\epsilon\left(\lambda^{\alpha} \bar{\lambda}_{\alpha}+\theta^{\alpha} r_{\alpha}\right)=\epsilon\left(\lambda^{\alpha} \bar{\lambda}_{\alpha}+n^{\alpha} r_{\alpha}\right)$ in (6.4) comes from the gauge-fixing term $-Q\left(n^{\alpha}\left(\bar{\Lambda}_{\alpha}-\epsilon \bar{\lambda}_{\alpha}\right)\right)$ in (4.5). Equations (6.4) and (6.5) are the gauge-fixed action and BRST operator of the non-minimal pure spinor formalism [47, 48] where the term $e^{-\int d^{2} z \epsilon\left(\lambda^{\alpha} \bar{\lambda}_{\alpha}+\theta^{\alpha} r_{\alpha}\right)}$ in $e^{-S}$ plays the role of a BRST-invariant regulator for integration over the zero modes of the pure spinors.

\section{Generalization to curved backgrounds}

The natural conjecture for generalizing the worldsheet reparameterization invariant action of (2.2) to a curved Type II target-space background is

$$
\begin{aligned}
S=\int d^{2} z(\operatorname{det} e)\left[\frac{1}{2}\left(g_{m n}(x)+b_{m n}(x)\right) \nabla x^{m} \bar{\nabla} x^{n}+w_{\alpha} \bar{\nabla} \lambda^{\alpha}+\widehat{w}_{\hat{\alpha}} \nabla \widehat{\lambda}^{\hat{\alpha}}\right. \\
+\Omega_{m}{ }^{n p}(x) \bar{\nabla} x^{m}\left(w \gamma_{n p} \lambda\right)+\widehat{\Omega}_{m}{ }^{n p}(x) \nabla x^{m}\left(\widehat{w} \gamma_{n p} \widehat{\lambda}\right) \\
+R_{m n p q}(x)\left(w \gamma^{m n} \lambda\right)\left(\widehat{w} \gamma^{p q} \widehat{\lambda}\right)+L^{\alpha} C_{\alpha}+\bar{\Lambda}_{\alpha} \lambda^{\alpha}+\widehat{L}^{\hat{\alpha}} \widehat{C}_{\hat{\alpha}} \\
\left.+\widehat{\bar{\Lambda}}_{\hat{\alpha}} \widehat{\lambda}^{\hat{\alpha}}+\frac{1}{4}\left(L \gamma^{m} \lambda\right)\left(\widehat{L} \gamma_{m} \widehat{\lambda}\right)\right]
\end{aligned}
$$

where $g_{m n}(x)$ and $b_{m n}(x)$ are the target-space metric and Kalb-Ramond field,

$$
\Omega_{m}^{n p}=\Gamma_{m}^{n p}+H_{m}^{n p} \text { and } \widehat{\Omega}_{m}^{n p}=\Gamma_{m}^{n p}-H_{m}^{n p}
$$

are the left and right-moving connections constructed as in the RNS action from the Christoffel connection $\Gamma_{m}^{n p}$ and the torsion $H_{m n p}=\partial_{[m} B_{n p]}, R_{m n p q}$ is the Riemann curvature tensor, $\gamma_{\alpha \beta}^{m}=E_{a}^{m}(x) \gamma_{\alpha \beta}^{a}$ where $a=0$ to 9 is a tangent-space index and $E_{a}^{m}$ is the target-space vierbein satisfying $\eta^{a b} E_{a}^{m} E_{b}^{n}=g^{m n}$, and

$$
C_{\alpha}=-\frac{1}{2} \nabla x^{m}\left(\gamma_{m} \lambda\right)_{\alpha} \quad \text { and } \quad \widehat{C}_{\hat{\alpha}}=-\frac{1}{2} \bar{\nabla} x^{m}\left(\gamma_{m} \widehat{\lambda}\right)_{\hat{\alpha}}
$$

are the twistor-like constraints in the curved background.

Surprisingly, the action of (7.1) has the same structure as the RNS worldsheet action if one replaces the left and right-moving pure spinor Lorentz currents $\left(w \gamma^{m n} \lambda\right)$ and $\left(\widehat{w} \gamma^{m n} \widehat{\lambda}\right)$ in (7.1) with the left and right-moving RNS Lorentz currents $\psi^{m} \psi^{n}$ and $\widehat{\psi}^{m} \widehat{\psi}^{n}$ and replaces the Lagrange multipliers $\left(L \gamma^{m} \lambda\right)$ and $\left(\widehat{L} \gamma^{m} \widehat{\lambda}\right)$ in $(7.1)$ with $\xi \psi^{m}$ and $\widehat{\xi} \widehat{\psi}^{m}$ where $\xi$ and $\widehat{\xi}$ are the RNS worldsheet gravitini and $\psi^{m}$ and $\widehat{\psi}^{m}$ are the RNS fermionic vectors. Just as the structure of the RNS action is determined by worldsheet supersymmetry, the structure of (7.1) is determined by the requirement that $C_{\alpha}$ and $\widehat{C}_{\hat{\alpha}}$ in (7.3) generate symmetries of the action.

Although the Ramond-Ramond background fields do not appear in (7.1), one expects that consistency of the gauge-fixing procedure will require that they appear in both the BRST transformations and in the gauge-fixed action. To be more specific, one needs to follow the procedure of $(5.3)$ and construct $\left(\theta^{\alpha}, p_{\alpha}\right)$ and $\left(\widehat{\theta}^{\hat{\alpha}}, \widehat{p}_{\hat{\alpha}}\right)$ variables in terms of the Fadeev-Popov ghosts and antighosts such that $\left(\theta^{\alpha}, p_{\alpha}\right)$ and $\left(\widehat{\theta}^{\hat{\alpha}}, \widehat{p}_{\hat{\alpha}}\right)$ are independent of the 
choice of patch of pure spinor space. It is expected that this construction will necessarily involve the Ramond-Ramond background fields and will imply equations of motion for all of the background fields. So instead of obtaining the equations of motion for the NSNS background fields from quantum worldsheet superconformal invariance as in the RNS formalism, it is conjectured that the equations of motion for all of the background supergravity fields (including the Ramond-Ramond fields) will be obtained in this formalism by requiring that the gauge-fixed action and BRST operator are independent of the choice of patch of pure spinor space.

For example, for the Ramond-Ramond plane-wave background, the classical action of $(7.1)$ is

$$
\begin{aligned}
S=\int d^{2} z(\text { dete })\left[\frac{1}{2}\right. & \nabla x^{m} \bar{\nabla} x_{m}+\frac{1}{2} \mu^{2}\left(\nabla x^{+}\right)\left(\bar{\nabla} x^{+}\right) x^{j} x^{j}+w_{\alpha} \bar{\nabla} \lambda^{\alpha}+\widehat{w}_{\hat{\alpha}} \nabla \widehat{\lambda}^{\hat{\alpha}} \\
& +\mu^{2}\left(x^{j} \bar{\nabla} x^{+}\left(w \gamma^{j+} \lambda\right)+x^{j} \nabla x^{+}\left(\widehat{w} \gamma^{j+} \widehat{\lambda}\right)+\left(w \gamma^{j+} \lambda\right)\left(\widehat{w} \gamma^{j+} \hat{\lambda}\right)\right) \\
& \left.+L^{\alpha} C_{\alpha}+\bar{\Lambda}_{\alpha} \lambda^{\alpha}+\widehat{L}^{\hat{\alpha}} \widehat{C}_{\hat{\alpha}}+\widehat{\bar{\Lambda}}_{\hat{\alpha}} \widehat{\lambda}^{\hat{\alpha}}+\frac{1}{4}\left(L \gamma^{m} \lambda\right)\left(\widehat{L} \gamma_{m} \widehat{\lambda}\right)\right]
\end{aligned}
$$

where $j=1$ to $8, x^{ \pm}=x^{0} \pm x^{9}$, and $\mu^{2}$ is the nonzero component $R_{+j+j}$ of the curvature. Since the constraints $C_{\alpha}$ and $\widehat{C}_{\hat{\alpha}}$ of (7.3) are classically conserved, the action of (7.4) is invariant under local symmetries analogous to the flat background symmetries of (3.1) and (3.2). But combining the fermionic ghosts and antighosts for these symmetries into unconstrained patch-independent variables, $\left(\theta^{\alpha}, p_{\alpha}\right)$ and $\left(\widehat{\theta}^{\hat{\alpha}}, \widehat{p}_{\hat{\alpha}}\right)$, is expected to be more complicated than in (5.3) and to require Ramond-Ramond coupling terms such as $\mu \int d^{2} z\left(p \gamma^{+1234} \widehat{p}\right)$ in the action. The complete consistency of this gauge-fixing procedure is expected to lead to the conformally invariant pure spinor action for the plane-wave background of [49].

For a general curved background, the gauge-fixing procedure of section 4 and construction of patch-independent $\left(\theta^{\alpha}, p_{\alpha}\right)$ and $\left(\widehat{\theta}^{\hat{\alpha}}, \widehat{p}_{\hat{\alpha}}\right)$ variables is expected to imply a gauge-fixed action and BRST operator which coincides with the pure spinor worldsheet action and BRST operator of [50]

$$
\begin{aligned}
& S=\int d^{2} z\left[\left(G_{M N}(x, \theta, \widehat{\theta})+B_{M N}(x, \theta, \widehat{\theta})\right) \partial Z^{M} \bar{\partial} Z^{N}+\ldots\right] \\
& Q=\int d z \lambda^{\alpha} d_{\alpha}, \quad \widehat{Q}=\int d \bar{z} \widehat{\lambda}^{\hat{\alpha}} \widehat{d}_{\hat{\alpha}}
\end{aligned}
$$

where $\left[G_{M N}, B_{M N}, \ldots\right]$ are the Type II supergravity superfields described in [50], $Z^{M}=$ $\left(x^{m}, \theta^{\alpha}, \widehat{\theta}^{\hat{\alpha}}\right)$ are the $\mathrm{N}=2 \mathrm{~d}=10$ superspace variables, and $p_{\alpha}$ and $\widehat{p}_{\hat{\alpha}}$ are the canonical momentum variables for $\theta^{\alpha}$ and $\widehat{\theta}^{\hat{\alpha}}$ defined by

$$
\begin{aligned}
& p_{\alpha}=d_{\alpha}-B_{\alpha M}\left(\partial Z^{M}-\bar{\partial} Z^{M}\right)-\Omega_{\alpha}{ }^{m n}\left(\lambda \gamma_{m n} w\right)-\widehat{\Omega}_{\alpha}{ }^{m n}\left(\widehat{\lambda} \gamma_{m n} \widehat{w}\right), \\
& \widehat{p}_{\hat{\alpha}}=\widehat{d}_{\hat{\alpha}}-B_{\hat{\alpha} M}\left(\partial Z^{M}-\bar{\partial} Z^{M}\right)-\Omega_{\hat{\alpha}}{ }^{m n}\left(\lambda \gamma_{m n} w\right)-\widehat{\Omega}_{\hat{\alpha}}{ }^{m n}\left(\widehat{\lambda} \gamma_{m n} \widehat{w}\right) .
\end{aligned}
$$

It would of course be very important to verify these conjectures for the curved Type II supergravity background. The first step would be to study the physical states in an 
open string background which should include both the super-Yang-Mills gluon and gluino. Since the gluino vertex operator is fermionic, it is absent from the reparameterization invariant action which only depends on bosonic worldsheet variables. This means that one should find non-trivial BRST cohomology at nonzero ghost number using the conventional definition of ghost number where the ghosts $\left(f^{\alpha}, g_{\alpha}\right)$ and antighosts $\left(m_{\alpha}, n^{\alpha}\right)$ carry ghost number +1 and -1 . After understanding how this works for the open superstring, it should be straightforward to generalize to the Type II superstring by taking the left-right product of two open superstrings.

\section{Acknowledgments}

I would like to thank Sergei Cherkis, Andrei Mikhailov, Warren Siegel, Cumrun Vafa, Edward Witten, and especially Nikita Nekrasov for useful discussions over the last several years, and CNPq grant 300256/94-9 and FAPESP grants 2009/50639-2 and 2011/11973-4 for partial financial support.

Open Access. This article is distributed under the terms of the Creative Commons Attribution License (CC-BY 4.0), which permits any use, distribution and reproduction in any medium, provided the original author(s) and source are credited.

\section{References}

[1] P. Ramond, Dual theory for free fermions, Phys. Rev. D 3 (1971) 2415 [inSPIRE].

[2] A. Neveu and J.H. Schwarz, Factorizable dual model of pions, Nucl. Phys. B 31 (1971) 86 [INSPIRE].

[3] F. Gliozzi, J. Scherk and D.I. Olive, Supersymmetry, supergravity theories and the dual spinor model, Nucl. Phys. B 122 (1977) 253 [INSPIRE].

[4] M.B. Green and J.H. Schwarz, Supersymmetrical dual string theory, Nucl. Phys. B 181 (1981) 502 [INSPIRE].

[5] M.B. Green and J.H. Schwarz, Covariant description of superstrings, Phys. Lett. B 136 (1984) 367 [INSPIRE].

[6] W. Siegel, Hidden local supersymmetry in the supersymmetric particle action, Phys. Lett. B 128 (1983) 397 [INSPIRE].

[7] N. Berkovits, Super Poincaré covariant quantization of the superstring, JHEP 04 (2000) 018 [hep-th/0001035] [INSPIRE].

[8] H. Gomez and C.R. Mafra, The closed-string 3-loop amplitude and S-duality, JHEP 10 (2013) 217 [arXiv: 1308.6567] [INSPIRE].

[9] C.R. Mafra, O. Schlotterer and S. Stieberger, Complete N-point superstring disk amplitude I. Pure spinor computation, Nucl. Phys. B 873 (2013) 419 [arXiv:1106.2645] [INSPIRE].

[10] H. Gomez and C.R. Mafra, The overall coefficient of the two-loop superstring amplitude using pure spinors, JHEP 05 (2010) 017 [arXiv:1003.0678] [INSPIRE].

[11] N. Berkovits, Quantum consistency of the superstring in $A d S_{5} \times S^{5}$ background, JHEP 03 (2005) 041 [hep-th/0411170] [INSPIRE]. 
[12] A. Mikhailov and S. Schäfer-Nameki, Perturbative study of the transfer matrix on the string worldsheet in $A d S_{5} \times S^{5}$, Adv. Theor. Math. Phys. 15 (2011) 913 [arXiv:0706.1525] [INSPIRE].

[13] L. Mazzucato, Superstrings in AdS, Phys. Rept. 521 (2012) 1 [arXiv:1104.2604] [InSPIRE].

[14] W. Siegel, Classical superstring mechanics, Nucl. Phys. B 263 (1986) 93 [INSPIRE].

[15] M. Matone, L. Mazzucato, I. Oda, D. Sorokin and M. Tonin, The superembedding origin of the Berkovits pure spinor covariant quantization of superstrings, Nucl. Phys. B 639 (2002) 182 [hep-th/0206104] [INSPIRE].

[16] Y. Aisaka and Y. Kazama, Origin of pure spinor superstring, JHEP 05 (2005) 046 [hep-th/0502208] [INSPIRE].

[17] N. Berkovits, Explaining the pure spinor formalism for the superstring, JHEP 01 (2008) 065 [arXiv: 0712.0324] [INSPIRE].

[18] N. Berkovits, Pure spinors, twistors and emergent supersymmetry, JHEP 12 (2012) 006 [arXiv: 1105.1147] [INSPIRE].

[19] N.A. Nekrasov, Lectures on curved beta-gamma systems, pure spinors and anomalies, hep-th/0511008 [INSPIRE].

[20] L.P. Hughston, The wave equation in even dimensions, in Further advances in twistor theory, volume 1, L.J. Mason at al. eds., Research Notes in Mathematics 231, Longman, U.K. (1990).

[21] L.P. Hughston, A remarkable connection between the wave equation and pure spinors in higher dimensions, in Further advances in twistor theory, volume 1, L.J. Mason at al. eds., Research Notes in Mathematics 231, Longman, U.K. (1990).

[22] L.P. Hughston and L.J. Mason, A generalized Kerr-Robinson theorem, Class. Quant. Grav. 5 (1988) 275 [INSPIRE].

[23] J.P. Harnad and S. Shnider, Isotropic geometry, twistors and supertwistors. 1. The generalized klein correspondence and spinor flags, J. Math. Phys. 33 (1992) 3197 [INSPIRE].

[24] J.P. Harnad and S. Shnider, Isotropic geometry and twistors in higher dimensions. 2: odd dimensions, reality conditions and twistor superspaces, J. Math. Phys. 36 (1995) 1945 [INSPIRE].

[25] P. Budinich and A. Trautman, The spinorial chessboard, Trieste Notes in Physics, Springer, Berlin Germany (1988).

[26] P. Budinich, From the geometry of pure spinors with their division algebras to fermion's physics, Found. Phys. 32 (2002) 1347 [hep-th/0107158] [INSPIRE].

[27] P. Furlan and R. Raczka, Intrinsic nonlinear spinor wave equations associated with nonlinear spinor representations, J. Math. Phys. 27 (1986) 1883.

[28] P. Furlan and R. Raczka, Nonlinear spinor representations, J. Math. Phys. 26 (1985) 3021.

[29] N. Berkovits and S.A. Cherkis, Higher-dimensional twistor transforms using pure spinors, JHEP 12 (2004) 049 [hep-th/0409243] [INSPIRE].

[30] R. Penrose, Twistor algebra, J. Math. Phys. 8 (1967) 345 [INSPIRE].

[31] E. Witten, Twistor-like transform in ten-dimensions, Nucl. Phys. B 266 (1986) 245 [INSPIRE]. 
[32] B.E.W. Nilsson, Pure spinors as auxiliary fields in the ten-dimensional supersymmetric Yang-Mills theory, Class. Quant. Grav. 3 (1986) L41 [InSPIRE].

[33] L.P. Hughston and W.T. Shaw, Real classical strings, Proc. Roy. Soc. Lond. A 414 (1987) 415 [INSPIRE].

[34] L.P. Hughston and W.T. Shaw, Classical strings in ten-dimensions, Proc. Roy. Soc. Lond. A 414 (1987) 423 [INSPIRE].

[35] A.K.H. Bengtsson, I. Bengtsson, M. Cederwall and N. Linden, Particles, superparticles and twistors, Phys. Rev. D 36 (1987) 1766 [inSPIRE].

[36] E. Sokatchev, Harmonic superparticle, Class. Quant. Grav. 4 (1987) 237 [InSPIRE].

[37] D.P. Sorokin, V.I. Tkach and D.V. Volkov, Superparticles, twistors and Siegel symmetry, Mod. Phys. Lett. A 4 (1989) 901 [inSPIRE].

[38] N. Berkovits, A supertwistor description of the massless superparticle in ten-dimensional superspace, Phys. Lett. B 247 (1990) 45 [INSPIRE].

[39] E. Bergshoeff, P.S. Howe, C.N. Pope, E. Sezgin and E. Sokatchev, Ten-dimensional supergravity from lightlike integrability in loop superspace, Nucl. Phys. B 354 (1991) 113 [INSPIRE].

[40] P.S. Howe, Pure spinors, function superspaces and supergravity theories in ten-dimensions and eleven-dimensions, Phys. Lett. B 273 (1991) 90 [INSPIRE].

[41] P.S. Howe, Pure spinors lines in superspace and ten-dimensional supersymmetric theories, Phys. Lett. B 258 (1991) 141 [Addendum ibid. B 259 (1991) 511] [InSPIRE].

[42] N. Berkovits, Covariant quantization of the superparticle using pure spinors, JHEP 09 (2001) 016 [hep-th/0105050] [INSPIRE].

[43] M. Cederwall, B.E.W. Nilsson and D. Tsimpis, Spinorial cohomology and maximally supersymmetric theories, JHEP 02 (2002) 009 [hep-th/0110069] [INSPIRE].

[44] N. Berkovits, Towards covariant quantization of the supermembrane, JHEP 09 (2002) 051 [hep-th/0201151] [INSPIRE].

[45] M. Cederwall, $D=11$ supergravity with manifest supersymmetry, Mod. Phys. Lett. A 25 (2010) 3201 [arXiv: 1001.0112] [INSPIRE].

[46] M. Cederwall and A. Karlsson, Loop amplitudes in maximal supergravity with manifest supersymmetry, JHEP 03 (2013) 114 [arXiv:1212.5175] [INSPIRE].

[47] N. Berkovits, Pure spinor formalism as an $N=2$ topological string, JHEP 10 (2005) 089 [hep-th/0509120] [INSPIRE].

[48] N. Berkovits and N. Nekrasov, Multiloop superstring amplitudes from non-minimal pure spinor formalism, JHEP 12 (2006) 029 [hep-th/0609012] [INSPIRE].

[49] N. Berkovits, Conformal field theory for the superstring in a Ramond-Ramond plane wave background, JHEP 04 (2002) 037 [hep-th/0203248] [INSPIRE].

[50] N. Berkovits and P.S. Howe, Ten-dimensional supergravity constraints from the pure spinor formalism for the superstring, Nucl. Phys. B 635 (2002) 75 [hep-th/0112160] [INSPIRE]. 